\title{
ERRATA
}

Senji Hoshi • Osamu Yamaguchi · Tomoaki Fujioka

Yoichi Arai · Yoshihiko Tomita • Tomonori Habuchi

Chikara Ohyama • Tadashi Suzuki • Seiichi Orikasa

The Tohoku Prostate Cancer Study Group

\section{A randomized comparative study of endocrine monotherapy and a combination of estramustine phosphate with the endocrine therapy in patients with untreated stage $D$ prostate cancer}

\section{Int J Clin Oncol (2006) 11: 303-308}

Errors appeared in the article cited above.

Tables 1-3 and Figures 1-3 should have appeared as follows:

S. Hoshi $(\bowtie)$

Department of Urology, Yamagata Prefectural Central Hospital,

1800 Aoyagi, Yamagata 990-2292, Japan

Tel. +81-23-685-2626; Fax +81-23-685-2608

e-mail: hoshi@ypch.gr.jp

O. Yamaguchi

Department of Urology, Fukushima Medical University School of Medicine, Fukushima, Japan

T. Fujioka

Department of Urology, Iwate Medical University School of

Medicine, Morioka, Japan

Y. Arai $\cdot$ S. Orikasa

Department of Urology, Tohoku University Graduate School of

Medicine, Sendai, Japan

Y. Tomita

Department of Urology, Yamagata University School of Medicine,

Yamagata, Japan

T. Habuchi

Department of Urology, Akita University School of Medicine, Akita, Japan

C. Ohyama $\cdot$ T. Suzuki

Department of Urology, Hirosaki University School of Medicine,

Hirosaki, Japan
Table 1. Distribution of patient characteristics

\begin{tabular}{|c|c|c|c|}
\hline Characteristics & $\begin{array}{l}\text { Endocrine } \\
+ \text { EMP }\end{array}$ & $\begin{array}{l}\text { Endocrine } \\
\text { alone }\end{array}$ & $\begin{array}{l}P \\
\text { value }\end{array}$ \\
\hline Randomized $(n)$ & 33 & 30 & \\
\hline Not eligible $(n)$ & 2 & 4 & \\
\hline Eligible $(n)$ & 31 & 26 & \\
\hline \multicolumn{4}{|l|}{ Age (years) } \\
\hline Mean \pm SE & $69.8 \pm 1.0$ & $70.0 \pm 1.2$ & \multirow[t]{2}{*}{0.9636} \\
\hline Median (range) & $70(57-78)$ & $69(60-79)$ & \\
\hline \multicolumn{4}{|c|}{ Endocrine therapy $(n)$} \\
\hline $\begin{array}{l}\text { LH-RH agonist } \\
\text { anti-androgen }\end{array}$ & 22 & 27 & \multirow{2}{*}{$0.2007^{\mathrm{b}}$} \\
\hline Others & 9 & 5 & \\
\hline \multicolumn{4}{|c|}{ Performance status $(n)$} \\
\hline 0 & 14 & 14 & \multirow{4}{*}{$0.4139^{\mathrm{b}}$} \\
\hline 1 & 11 & 10 & \\
\hline 2 & 4 & 0 & \\
\hline 3 & 2 & 2 & \\
\hline \multicolumn{4}{|c|}{ Pathological grade $(n)$} \\
\hline Well diff. & 2 & 4 & \multirow{3}{*}{$0.7793^{\mathrm{b}}$} \\
\hline Moderately diff. & 19 & 13 & \\
\hline Poorly diff. & 10 & 9 & \\
\hline \multicolumn{4}{|l|}{ EOD grade $(n)$} \\
\hline 0 & 2 & 0 & \multirow{4}{*}{$0.1183^{\mathrm{b}}$} \\
\hline 1 & 16 & 10 & \\
\hline 2 & 10 & 11 & \\
\hline 3 & 3 & 5 & \\
\hline \multicolumn{4}{|l|}{ Metastatic sites $(n)$} \\
\hline Lung & 0 & 1 & \\
\hline Bone & 29 & 27 & \\
\hline Lymph node & 11 & 13 & \\
\hline Liver & 0 & 1 & \\
\hline \multicolumn{4}{|l|}{ Pretreatment } \\
\hline PSA (ng/ml) & & & \multirow{3}{*}{$0.4748^{\mathrm{a}}$} \\
\hline Mean \pm SE & $673.8 \pm 224.8$ & $946.6 \pm 315.7$ & \\
\hline Median (range) & $171.2(2.3-4800)$ & $230(1.8-5930)$ & \\
\hline \multicolumn{4}{|l|}{$\begin{array}{l}\text { Follow-up time } \\
\text { (weeks) }\end{array}$} \\
\hline Mean \pm SE & $94.3 \pm 13.4$ & $96.4 \pm 14.4$ & \multirow[t]{2}{*}{$0.8552^{\mathrm{a}}$} \\
\hline Median (range) & $76.3(8.3-228.1)$ & $92.3(1.4-243.7)$ & \\
\hline
\end{tabular}

${ }^{\mathrm{a}} t$-test

${ }^{\mathrm{b}}$ Mann-Whitney test 
Table 2. Analysis of overall survival in both groups

\begin{tabular}{|c|c|c|c|c|c|}
\hline \multirow[t]{2}{*}{ Characteristics } & \multicolumn{2}{|c|}{ Number of patients $(n)$} & \multicolumn{2}{|c|}{ Median survival (weeks) } & \multirow{2}{*}{$\begin{array}{l}P \text { value } \\
\text { (log-rank test) }\end{array}$} \\
\hline & $\mathrm{E}+\mathrm{EMP}$ & $\mathrm{E}$ & $\mathrm{E}+\mathrm{EMP}$ & $\mathrm{E}$ & \\
\hline \multicolumn{6}{|l|}{ PS } \\
\hline 0 & 13 & 13 & Not reached & Not reached & 0.2834 \\
\hline $1-3$ & 13 & 10 & Not reached & 133 & 0.0422 \\
\hline \multicolumn{6}{|l|}{ Age (years) } \\
\hline$<74$ & 17 & 16 & Not reached & 121 & 0.0259 \\
\hline$\geqq 74$ & 9 & 7 & Not reached & Not reached & 0.7322 \\
\hline \multicolumn{6}{|c|}{ Baseline PSA (ng/ml) } \\
\hline$\leqq 20$ & 2 & 4 & Not reached & Not reached & 0.4945 \\
\hline$>20$ & 24 & 19 & Not reached & 133 & 0.0373 \\
\hline \multicolumn{6}{|l|}{ EOD grade } \\
\hline $0-2$ & 23 & 19 & Not reached & Not reached & 0.0529 \\
\hline $3-4$ & 3 & 4 & Not reached & Not reached & 0.8140 \\
\hline \multicolumn{6}{|l|}{ Pathological grade } \\
\hline Well & 2 & 3 & Not reached & Not reached & 0.1308 \\
\hline Moderate, poor & 24 & 20 & Not reached & 166 & 0.0290 \\
\hline \multicolumn{6}{|c|}{ PSA evaluation at 12 weeks } \\
\hline CR & 14 & 14 & Not reached & Not reached & 0.2134 \\
\hline PR & 9 & 8 & Not reached & 102 & 0.0262 \\
\hline
\end{tabular}

E, endocrine therapy; EMP, estramustine phosphate

Table 3. Incidence of side effects

\begin{tabular}{lll}
\hline & $\mathrm{E}+\mathrm{EMP}(n=31)$ & $\mathrm{E}(n=26)$ \\
\hline Gastrointestinal toxicity & $4(2)$ & 1 \\
Liver dysfunction & 4 & 3 \\
Cardiovascular disorder & $5(1)$ & $1(1)$ \\
Anemia & 3 & 0 \\
Others & 3 & 3 \\
Total number of cases & $14 / 31(45.2 \%)$ & $7 / 26(26.9 \%)$
\end{tabular}

E, endocrine therapy; EMP, estramustine phosphate

Numbers in parentheses indicate grade 3 or higher side effects

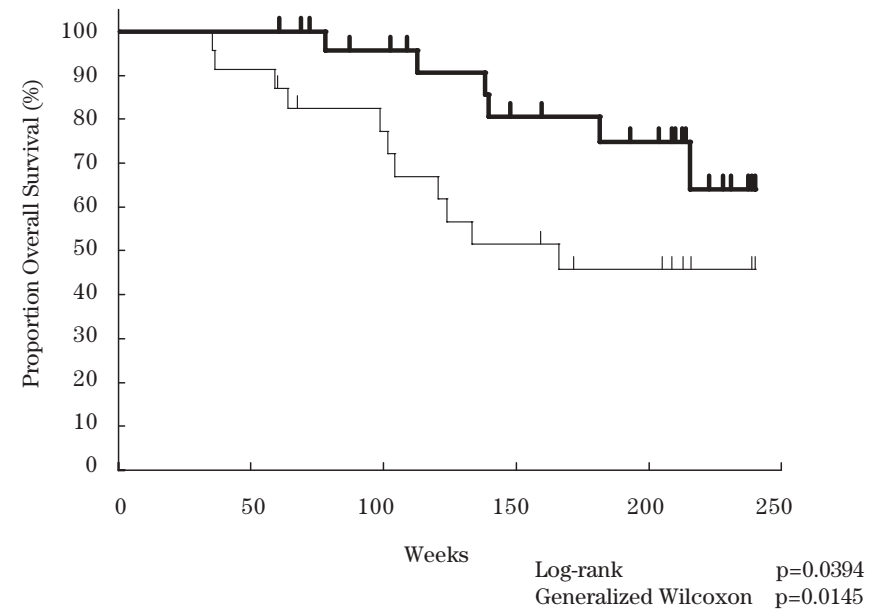

Fig. 2. Kaplan-Meier estimate of overall survival. The median time to death was not reached in the combination therapy group (thick line) vs 166 weeks in the endocrine monotherapy group (thin line). Log-rank, $P$ $=0.0394$; generalized Wilcoxon test, $P=0.0145$

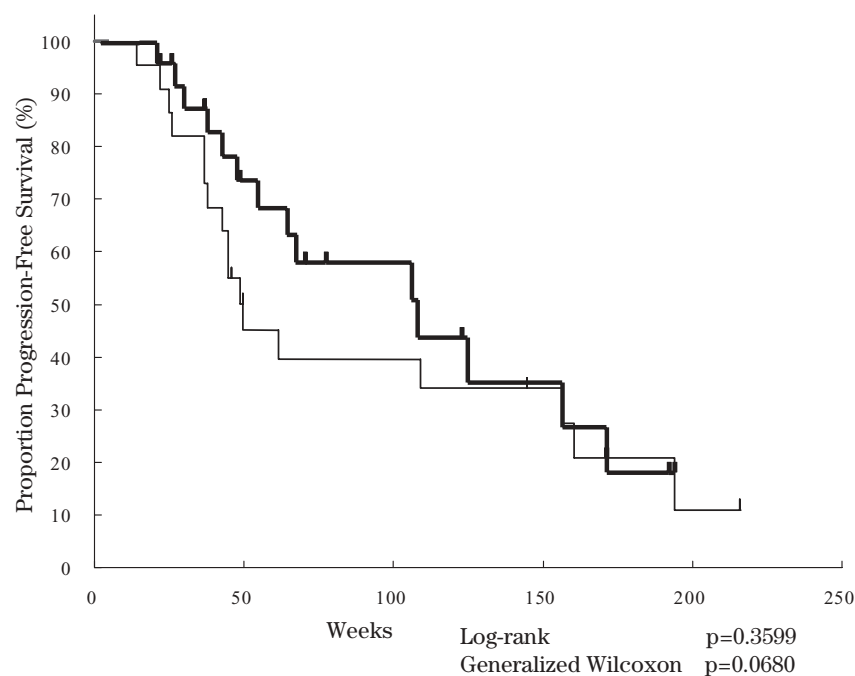

Fig. 1. Kaplan-Meier estimate of progression-free survival. The median time to progression was 107 weeks in the group with a combination of endocrine therapy and EMP (thick line) vs 48 weeks in the endocrine monotherapy group (thin line). Log-rank, $P=0.3599$; generalized Wilcoxon test, $P=0.0680$

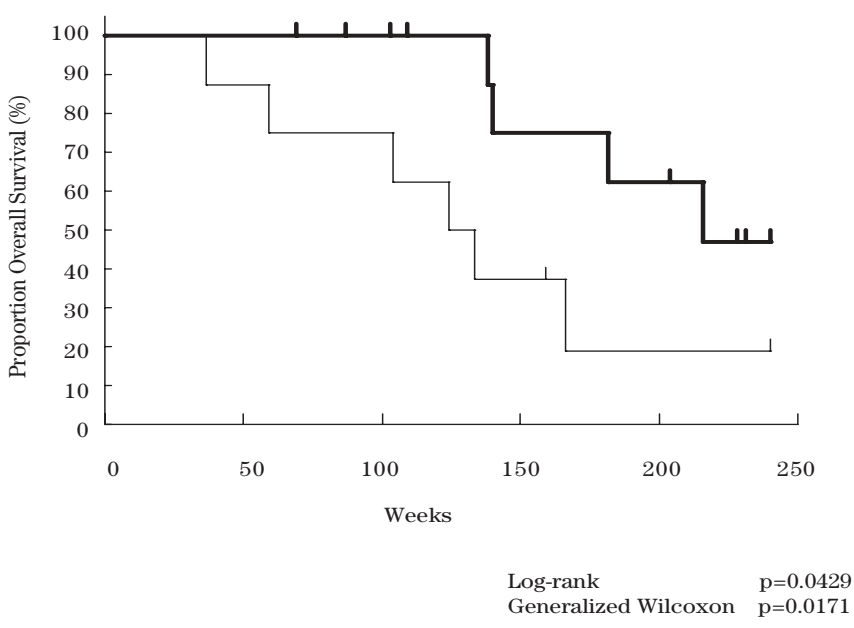

Fig. 3. Kaplan-Meier estimate of overall survival in patients who had three poor prognosis factors (performance status [PS] of 1 to 3 , pretreatment prostate-specific antigen [PSA] level of more than $20 \mathrm{ng} / \mathrm{ml}$, and moderately or poorly differentiated carcinoma). The median time to death was 215 weeks in the combination therapy group (thick line) vs 133 weeks in the endocrine monotherapy group (thin line). Log-rank, $P=0.0429 ;$ generalized Wilcoxon test, $P=0.0171$ 